\title{
Spatial Correlation Characterization for UWB Indoor Channel Based on Measurements
}

\author{
H. Chaibi ${ }^{1}$, R. Saadane ${ }^{2}$, My A. Faqihi ${ }^{1}$, and M. Belkasmi ${ }^{1}$ \\ ${ }^{1}$ Ecole Nationale Supérieure d'Informatique et d'Analyse des Systèmes, Rabat, \\ Suissi, Rabat, Maroc \\ 2 SIR2C2S/LETI-EHTP, Ecole Hassania des Travaux Publiques, Casablanca Maroc \\ \{has.chaabi, rachid.saadane\}@gmail.com, \\ ffaqihi, belkasmi\}aensias.ma
}

\begin{abstract}
The major aim of this paper is to present a statistical model for small scale fading and spatial correlation evaluation for indoor UWB channel. A some distributions are suggested for small scale fading statistics where the number of scatterers is unknown. Also, the impact of a number of system parameters on spatial correlation at the receiver is evaluated. The complex correlation coefficient decays slowly with distance under line-of-sight, but decreases quickly under non Line of sight.
\end{abstract}

Index Terms: UWB Channel Characterization Modeling, Measurements, Small Scale Statistics, Amplitude and Phase Distribution.

\section{Introduction}

Due to current developments in digital consumer electronics technology, Ultra Wideband (UWB) is becoming extra attractive for low cost personal communication applications. UWB systems are now emerging across a diversity of commercial and military applications, including communications, radar, geolocation, and medical. First generation commercial wireless UWB creations are anticipated to be extensively deployed almost immediately. This has been fueled by a command for high frequency consumption and a large number of users requiring simultaneous multidimensional high data rate access for applications of wireless internet and e-commerce [2,6, 10, 11].

UWB systems are often defined as systems that have a relative bandwidth larger than $25 \%$ and/or an absolute bandwidth of more than $500 \mathrm{MHz}$ (FCC). The UWB systems using large absolute bandwidth, are robust to frequency-selective fading, which has significant implications on both design and implementation [10]. Among its significant characteristics, the UWB technology are low power devices, exact localization, high multi-path resistance, low complexity hardware structures and carrier-less architectures [8, 9, 14]. As well, the spreading of the information over a very huge frequency range decreases the spectral density and makes UWB technology deployment compatible with existing systems.

As first step in for designing and implementing any wireless communication system, channel measurements and modeling are a basic necessity. Several theoretical and 
practical studies, have shown an extreme difference with respect to narrow-band channels [15]. In the area of UWB channel modeling, the researchers are interested to characterize the path loss law, shadowing, multi-path delay spread, coherence bandwidth, average multi-path intensity profile and received amplitude distribution of the multipath components... But, there is no universal UWB channel model proposed.

The contribution of this paper is a simple modeling of small scale fading and simple empirical model relating correlation coefficient to distance of UWB indoor channel. The propagation scenarios deal with both Line-of-Sight (LOS) and Non-Line-of-Sight (NLOS) situations. We have assumed two hypotheses, the first one is that the indoor channel is considered to be time invariant because the transmitter and the receiver are static and no motions take place in the channel. The second one is that the signal excitation is assumed to be close to an ideal Dirac-Delta impulse which means that the received signal can be seen as a good approximation of the channel impulse response.

The rest of the paper is organized as follows. In Section 2 presents our measurement specification and set-up. Section 3 presents channel model and Statistical distributions. The Section 4 describes our spatial correlation channel analysis and results. Finally, conclusions are provided in Section 5

\section{Measurement Specification and Set-Up}

Measurements are performed at spatially different locations under both Line of Sight (LOS) and Non Line of Sight (NLOS). These are carried out in Eurecom Mobile Communication Laboratory, which has a typical laboratory environment (radio frequency equipment, computers, tables, chairs, metallic cupboard, glass windows,...) with plenty of reflective and diffractive objects, rich in reflective and diffractive objects. For the NLOS case, a metallic plate is positioned between the transmitter and the receiver [6]. We have complete database of 4000 channel frequency responses corresponding to different scenarios with a transmitter-to-receiver distance varying distance varying from 1 meter to 14 meters. The Electrical specifications of the used antennas in this work are [12]:

- Frequency Range: $3.1-10.0 \mathrm{GHz}$.

- Gain: $4.4 \mathrm{dBi}$ peak at $4.5 \mathrm{GHz}$.

- VSWR 1 - $2.0: 1$ : across $3.6-9.1 \mathrm{GHz}$.

- Polarization: Linear.

- Radiation Pattern: Azimuth Omni-directional.

- Feed Impedance: $50 \Omega$ (Ohms) Unbalanced.

The Mechanical Specification [12]:

- Antenna Element: $0.54 \times 0.63 \times 0.12$ in $13.6 \times 16.0 \times 3.0 \mathrm{~mm}$

- Assembly PCB: $1.03 \times 0.73 \times .04$ in $26.2 \times 18.5 \times 1.0 \mathrm{~mm}$

- Area of PCB that is Ground: $0.48 \times 0.73$ in $12.2 \times 18.5 \mathrm{~mm}$

- Antenna Element Weight: 0.5 g

${ }^{1}$ Voltage Standing Wave Ratio. 
The antennas presents a VSWR varying from 2 to 5 for example an efficiency about $82 \%$ at $5.2 \mathrm{GHz}$. From the Fig. 2 in [6] we see a plate response over frequency range, this a very important characteristics of UWB antenna, we recommend reference [13] to learn about analysis of the antennas impact on the channel measurements.

\subsection{Configuration and Set-Up}

In our spatial correlation analysis we have used a virtual antenna arrays at the receiver, with a fixed transmitter. A manual controlled positioning grid is used to scan the area by $1 \mathrm{~cm}$. With respect of this scheme, receiving linear grid is synthesized in both broadside and end-fire configurations with minimum inter-element spacing $d=1 \mathrm{~cm}$, maximum aperture width $D=100 \mathrm{~cm}$, and up to $N_{d}=40$ elements, as depicted in Fig. 1 Due to aperture synthesis and the calibration processes, this analysis does not include the effects of antenna coupling, and the scope of the present conversation is limited to spatial correlation analysis, for LOS and NLOS configurations.

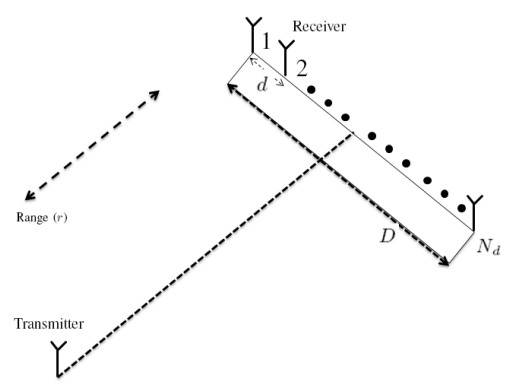

Fig. 1. General configuration of Eurecom UWB channel Measurement

\subsection{Channel and Time Stationarity}

The time stationarity of the channel is a obligation for the validity of the array synthesis approach, and is ensured by completely immobilizing the measurement environment, as established in [16] for example. This is in line with previous studies demonstrating the time stationarity of indoor office and residential environments [15, 16]. A different operating scenario, such as an outdoor UWB channel, may not reveal time stationarity, and the spatial correlation characteristics may then be different in time [16]. Several indoor small-office environments with size of the order of $10 \mathrm{~m} \times 6 \mathrm{~m}$ and strong number of multi-paths are detected. The settings within the laboratories are varied, and the transmitter receiver separation ranges from 1 to $6 \mathrm{~m}$. Representing the receiving antenna position on the Cartesian measurement grid by the cross-range, $x$, and range, $r$, components, and the corresponding sets of locations by $X$ and $R$, respectively, we can express the Complex Channel Transfer Function (CCTF) as [16]

$$
T(x, r, f)=\sum_{n=1}^{F} A(x, r, n) \exp ^{j \theta(x, r, n)} \delta(f-n \Delta f)
$$


where $r \in R$ is the range location, $x \in X$ is the cross-range location, $A$ is the amplitude and $\theta$ is the phase. Under this representation, a variety of values of $x \in X$, with $r$ being constant, define a broadside array.

In this work, the spatial correlation coefficient, $\rho$, is calculated from the set of measured channel responses in the frequency domain. The receive correlation coefficient signifies the statistical correlation between the signals received at two different locations after being emitted by the same transmitter's position, at the same time as the transmit correlation is the converse quantity, i.e. the correlation with two transmitters and one receiver. Consider two CCTFs, $T_{1}=T\left(r_{1}, x_{1}, f\right)$ and $T_{2}=T\left(r_{2}, x_{2}, f\right)$, measured at locations $\left(r_{1}, x_{1}\right)$ and $\left(r_{2}, x_{2}\right)$, respectively, separated by distance, $d$, given by

$$
d=\sqrt{\left(r_{1}-r_{2}\right)^{2}+\left(x_{1}-x_{2}\right)^{2}} .
$$

The degree of likeness between these two CCTFs can be predicted in terms of their cross-correlation. We note that the CCTF is a random process as the frequency-domain fading coefficients are stochastic.

\section{Proposed Statistical Distributions Description}

The multipath indoor radio propagation channel impulse response on time domain is normally molded as a complex low-pass equivalent impulse response. To characterize the probability density function of the power variations in frequency domain $(H(f))$ we plot the histogram's measurement data [13]. The power variations are fitted with an analytical probability density function (pdf) approximation, namely a Weibull pdf. The general formula for the Weibull pdf is given by:

$$
f(z)=\frac{\gamma}{\alpha}\left(\frac{z-\mu}{\alpha}\right)^{(\gamma-1)} \exp \left\{-\left(\frac{z-\mu}{\alpha}\right)^{\gamma}\right\}
$$

where $\alpha, \gamma, \mu \in R, \alpha, \gamma>0$ and $z \geq \mu, \alpha$ is the scale parameter, $\gamma$ is the shape parameter, and $\mu$ is the location parameter.

The Probability density function Student's t-distribution has the probability density function:

$$
f(z)=\frac{\Gamma\left(\frac{\nu+1}{2}\right)}{\sqrt{\nu \pi} \Gamma\left(\frac{\nu}{2}\right)}\left(1+\frac{z^{2}}{\nu}\right)^{-(\nu+1) / 2}
$$

where $\nu$ is the number of degrees of freedom and $\Gamma$ is the Gamma function. The Rice law, The probability density function is:

$$
f(z \mid \nu, \sigma)=\frac{z}{\sigma^{2}} \exp \left[-\frac{\left(z^{2}+\nu^{2}\right)}{2 \sigma^{2}}\right] I_{0}\left(\frac{x \nu}{\sigma^{2}}\right)
$$

where $I_{0}(z)$ is the modified Bessel function of the first kind with order zero. When $\nu=0$, the distribution reduces to a Rayleigh distribution. 


\subsection{Evaluation}

About 2000 LOS measurements were used to qualifying the adequate distribution. Testing the results against the Weibull $t$ distribution in LOS, the Rice and the Student's models, we conclude as follows: For the amplitude distribution we found the Weibull pdf to have the best (highest) value of log likelihood score in about $70 \%$ of the measurements. The same results are founded for NLOS. For the phases distribution we have founded that is very fitted by a non parametric distribution with normal kernel for both LOS and NLOS cases see Figures 2 and 3 .

\section{Spatial Correlation}

Now, the correlation coefficient, $\rho$, between two complex random variables $u(\xi)$ and $v(\xi)$ can be evaluated using the general expression [16]

$$
\rho(u, v)=\frac{E(u v \dagger)-E(u) E(v \dagger)}{\sigma_{u} \sigma_{v}}
$$

where $E($.$) denotes expectation, \dagger$ denotes conjugation,

$$
\sigma_{u}=\sqrt{E\left\{|u|^{2}\right\}+|\{E(u)\}|^{2}} .
$$

and $\sigma_{v}$ is defined in a similar manner. In order to calculate the complex correlation coefficient, $\rho_{a}$, for the UWB channel using the CTFs, we use (6) and (7) with $u=H_{1}$, $v=H_{2}$ and $\xi=f$. The envelope correlation, $\rho_{e}$, and power correlation, $\rho_{p}$, defined in [16], provide alternative definitions of the correlation coefficient. However, $\rho_{e}$ and $\rho_{p}$ do not make use of the phase information in the complex CTFs. To calculate $\rho_{e}$, we put $u=\left|H_{1}\right|$ and $v=\left|H_{2}\right|$ in (6) and (6), while for $\rho_{p}$, we use $u=\left|H_{1}\right|^{2}$ and $v=\left|H_{2}\right|^{2}$. The approximation

$$
\rho_{e} \approx \rho_{p} \approx\left|\rho_{a}\right|^{2} .
$$

holds for Rayleigh-faded narrowband wireless channels [17], but not necessarily for other fade distributions. We evaluate these three types of correlation for UWB channels, analyzing the effect of the spatial offset, $d$. If the channel is asymptotically isotropic in the horizontal plane, the correlation becomes a two dimensional Bessel function of $d$ with contours circularly symmetric about the point of reference [16]. Fortunately, indoor UWB channels typically show large angular spreads [16], which is also reflected in our measurements.

\subsection{Correlation Evaluation}

This part will present the analysis to the spatial correlation characteristics as a function of range (distance), which signifies the performance of end fire arrays [16]. In order to achieve this, we calculate the expectation, over the measurement set, of the spatial correlation coefficient for a given range offset. The latter, in turn, is evaluated in terms of the correlation coefficient between the central element of the $x^{\text {th }}$ column of the 

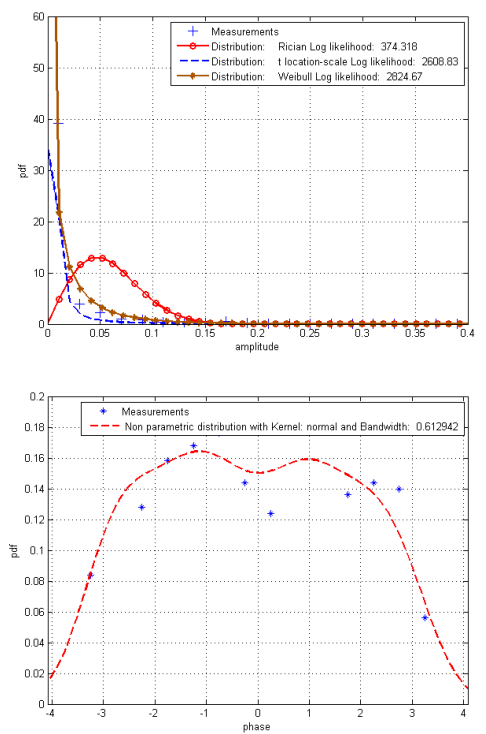

Fig. 2. Amplitude (left) and Phase (right) histogram together with the appropriate probability density functions for LOS case
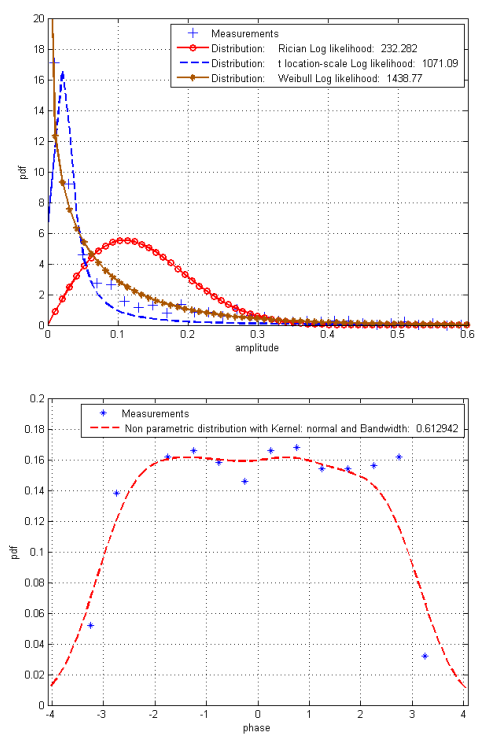

Fig. 3. Amplitude (left) and Phase (right) histogram together with the appropriate probability density functions for NLOS case 
spatial measurement grid and the other elements in that column. The mean correlation coefficient magnitudes thus obtained are shown in Fig. 4. It is observed that $\rho_{e} \approx \rho_{p}$ for all considered offset $d$. The range correlation has a broader main lobe support compared with the cross-range correlation. The first null of $\rho_{e}$ and $\rho_{p}$ is obtained at $d=20 \mathrm{~cm}$ approx. $\left(d=15 \mathrm{~cm}\right.$ approx. is reported in [16]), while the $\rho_{e}=0.5$ threshold is crossed at $d=9 \mathrm{~cm}(d=7 \mathrm{~cm}$ approx. is reported in [16]).
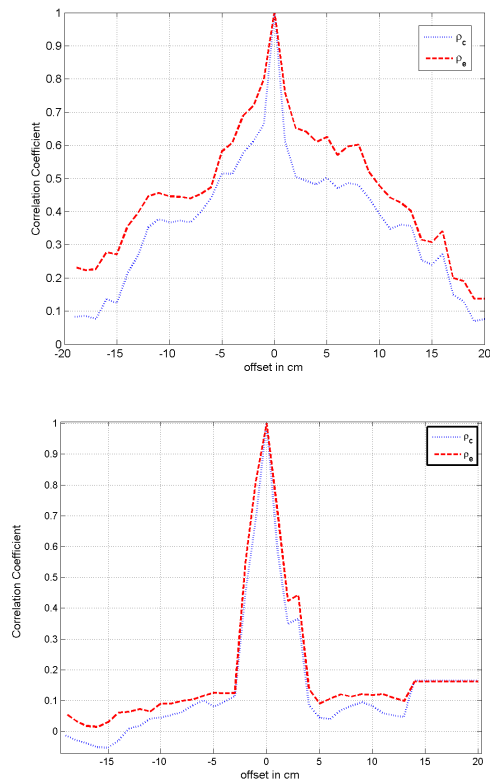

Fig. 4. The spatial correlation magnitude verses the spatial offset in LOS (left) and NLOS (right). The insets show the correlation for the corresponding LOS and NLOS channels. The complex $\left(\rho_{c}\right)$ and envelope $\left(\rho_{e}\right)$ correlation values are shown.

\section{Conclusion}

In this work, we have presented a simple empirical model for statistical small scale fading of UWB indoor channel with unknown number of scatters. The results shown that the channel small scale fading can be fitted very well by Weibull distribution for LOS and NLOS. Also, the analysis of correlation coefficient shows that the the complex correlation decays less rapidly with distance under LOS, but decreases rapidly under NLOS.

\section{References}

1. First report and order, revision of part 15 of the commission's rules regarding ultra-wideband transmission systems. FCC, ET Docket 98-153, February 14 (2002)

2. Ghassemzadeh, S.S., Jana, R., Rice, C.W., Turin, W., Tarokh, V.: Measurement and modeling of an ultra-wide bandwidth indoor channel. IEEE Trans. Commun. 52(10), 1786-1796 (2004) 
3. Molisch, A.F.: Ultrawideband propagation channels-theory, measurement,and models. IEEE Transaction Vehicular Technology (2005) (invited paper)

4. Cassioli, D., Win, M.Z., Molisch, A.F.: The ultra-wide bandwidth indoor channel: From statistical study to simulations. IEEE J. Select. Areas Commun. 20(6), 1247-1257 (2002)

5. Kunisch, J., Pamp, J.: Measurement results and modeling aspects for the UWB radio channel. In: Proc. UWBST, pp. 19-23 (May 2002)

6. Saadane, R., Menouni, A., Knopp, R., Aboutajdine, D.: Empirical eigenanalysis of indoor ULB propagation channels. In: IEEE Global Telecommunications Conference, November 29-December 3 (2004)

7. Molisch, A.F., Kannan, B., Chnog, C.C., Emami, S., Karedal, A., Kunisch, J., Shantz, H., Schuster, U., Siwiak, K.: IEEE 802.15.4a Channel model - final Report. IEEEE 802.15-040662-00-004a, San Antonio, TX, USA (November 2004)

8. Menouni Hayar, A., Knopp, R., Saadane, R.: Subspace analysis of indoor UWB channels. EURASIP Journal on Applied Signal Processing, Special Issue on UWB - State of the Art 2(3), 287-295 (2005)

9. Saadane, R., Menouni Hayar, A., Knopp, R., Aboutajdine, D.: On the estimation of the degrees of freedom of in-door UWB channel. In: VTC Spring 2005, May 29-June 1 (2005)

10. Saadane, R., Aboutajdine, D., Menouni Hayar, A., Knopp, R.: UWB Channel and Degrees of Freedom Evaluations. International Journal on Wireless and Optical Communications Special Issus in Ultra Wide, April 17 (2007)

11. Saadane, R., Aboutajdine, D., Menouni Hayar, A.: Ultra Widebandwidth Large and Small scale Characterization With Different Environments. In: ICTIS 2007 Conference, Fez, Morocco, April 3-5 (2007)

12. (2010), http: / /www. skycross.com

13. Saadane, R., El Aroussi, M., Hayar, A., Aboutajdine, D.: UWB Channel Modelling, Indoor Propagation: Statistical model Based on Large and Small Scales Analysis. IJSC International Journal of Computational Science, 1992-6669 (Print) 1992-6677 (2008)

14. Keignart, J., Pierrot, J.B., Danièle, N., Álvarez, Á., Lobeira, M., García, J.L., Valera, G., Torres, R.P.: Radio channel sounding results and model, Deliverable number: D31, IST-200132710-U.C.A.N

15. Chong, C.-C., Kim, Y., Lee, S.-S.: Statistical characterization of the UWB propagation channel in various types of high-rise apartments. In: Wireless Communications and Networking Conference, pp. 944-949 (March 2005)

16. Malik, W.Q.: Spatial correlation in ultrawideband channels. IEEE Trans. Wireless Commun. 7(2), 604-610 (2008)

17. LaMaire, R.O., Zorzi, M.: Effect of correlation in diversity systems with Rayleigh fading, shadowing, and power capture. IEEE J. Select. Areas Commun. 14(3) (April 1996) 